\title{
Whole Exome Sequencing Identified Novel Mutation in SUOX Gene Causes Extremely Rare Autosomal Recessive Isolated Sulfite Oxidase Deficiency
}

\section{Rui Zhang}

Shenzhen Baoan Shajing People's Hospital: Shenzhen Baoan Second People's Hospital

\section{Yajing Hao}

Shenzhen Baoan Shajing People's Hospital: Shenzhen Baoan Second People's Hospital

\section{Ying Xu}

Shenzhen Baoan Shajing People's Hospital: Shenzhen Baoan Second People's Hospital

Jiale Qin

Zhejiang University School of Medicine

\section{Yanfang Wang}

Shenzhen Baoan Shajing People's Hospital: Shenzhen Baoan Second People's Hospital

\section{Subrata Kumar Dey}

West Bengal University of Technology: Maulana Abul Kalam Azad University of Technology

\section{Chen Li}

Zhejiang University School of Medicine

\section{Huilin Wang}

Shenzhen Baoan Shajing People's Hospital: Shenzhen Baoan Second People's Hospital

Santasree Banerjee ( $\square$ santasree.banerjee@yahoo.com )

Jilin University https://orcid.org/0000-0003-2481-4562

\section{Research}

Keywords: ISOD syndrome, SUOX gene, novel variant, compound heterozygous, loss-of-function

Posted Date: September 10th, 2020

DOI: https://doi.org/10.21203/rs.3.rs-74199/v1

License: (c) (i) This work is licensed under a Creative Commons Attribution 4.0 International License. Read Full License 


\section{Abstract}

Background: Isolated sulfite oxidase deficiency (ISOD) is the rarest types of life-threatening neurometabolic disorders characterized by neonatal intractable seizures and severe developmental delay with an autosomal recessive mode of inheritance. ISOD is extremely rare and only 29 mutations have been identified and reported worldwide. Germline mutation in SUOX gene causes ISOD.

Results: Here, we investigated a 5-days old Chinese girl, presented with intermittent tremor or seizures of limbs, neonatal encephalopathy, subarachnoid cyst and haemorrhage, dysplasia of corpus callosum, neonatal convulsion, respiratory failure, cardiac failure, hyperlactatemia, severe metabolic acidosis, hyperglycemia, hyperkalemia, moderate anemia, atrioventricular block and complete right bundle branch block. Karyotype and chromosomal microarray analysis were performed and identified no chromosomal abnormalities in the proband. Whole exome sequencing was performed for the proband. Sanger sequencing was performed for the proband and her family members. Whole exome sequencing identified a novel heterozygous deletion (c.1406_1421delCCTGGCAGGTGGCTAA) and a previously reported heterozygous substitution (c.1200C>G) in exon 6 of the SUOX gene in the proband. The novel heterozygous deletion leads to frameshift ( $p$.Thr469Serfs*20) which results into the formation of a truncated sulfite oxidase of 488 amino acids. The substitution leads to a premature stop codon (p.Tyr400*) followed by the formation of a truncated sulfite oxidase of 399 amino acids. Hence, both the variants are loss-of-function variants. The proband's father and mother is carrying the substitution and deletion in a heterozygous state respectively. These two variants were not identified in the elder brother of the proband as well as in the 100 healthy individuals.

Conclusion: Here, we reported the first variant of SUOX gene associated ISOD in Chinese population. Our study not only expand the mutational spectrum of SUOX gene associated ISOD, but also strongly suggested the application of whole exome sequencing for identifying the novel disease-causing mutation in candidate genes.

\section{Background}

Isolated sulfite oxidase deficiency (ISOD) [MIM\# 272300] is one of the rarest types of neurometabolic disorders usually manifested with severe neurometabolic impairment and developmental delay [1]. ISOD patients with neonatal age of onset were usually characterized by very severe clinical phenotype which often leads to their death at their infancy [2]. Patients with ISOD were often presented with neonatal seizures, difficulties in feeding, high-pitched crying with irritation, severe developmental delay, abnormalities in muscle tone and movements [2]. Choreoathetosis and dystonia were often found in the patients with ISOD [3]. In later stages, ectopia lentis is also identified in ISOD patients [2]. ISOD patients were also manifested with biochemical abnormalities, often included excess secretion of sulfites and Ssulfocysteine through urine and low level of cysteine in plasma [3]. In addition, neuroimaging of the ISOD patients usually showed diffuse cortical swelling which gradually and progressively leads to the multicystic encephalomalacia, mimicking hypoxic-ischemic encephalopathy $[3,4]$. In patients with ISOD, 
soon after birth, neuroimaging usually found hypoplastic corpus callosum and cerebral infraction, cystic supratentorial white matter degeneration, with signal intensity changes in bilateral basal ganglia and thalami $[5,6]$. ISOD patients with neonatal disease onset were often died soon after birth or during infancy [2]. However, ISOD patients, those who are not died at their neonatal stage, usually manifested with cerebral palsy, severe developmental delay and encephalomalacia later.

Germline mutation in SUOX gene causes Isolated sulfite oxidase deficiency (ISOD). SUOX gene is located at the long arm of the chromosome 12 (12q13.2) and encodes sulfite oxidase consisting of 545 amino acids. Sulfite oxidase is located in the intermembrane space of mitochondria. Structurally, sulfite oxidase is consisting of two identical subunits (homodimer) and each subunit comprises of a N-terminal heme co-factor binding domain and a C-terminal molybdopterin-binding domain. Firstly, for obtaining catalytic enzymatic activity, sulfite oxidase interacts and bind with a molybdenum-containing cofactor. Then, it catalyzes the transformation of sulfite $\left(\mathrm{SO}_{3}{ }^{2-}\right)$ to sulphate $\left(\mathrm{SO}_{4}{ }^{2-}\right)$ by oxidation via cytochrome $\mathrm{c}$ through which the oxidative degradation of the sulfur-containing amino acids (cysteine and methionine) has been taken place [7]. Germline mutations in SUOX gene leads to the formation of partial or complete nonfunctional sulfite oxidase, followed by the impairment of the oxidative degradation of sulfite $\left(\mathrm{SO}_{3}{ }^{2-}\right)$ to sulfate $\left(\mathrm{SO}_{4}{ }^{2-}\right)$. Partial or complete deficiency of sulfite oxidase leads to fatal neurological abnormalities at neonatal stage.

The patients with ISOD usually manifested with either fatal early-onset form presented with seizures, severe psychomotor retardation and death or late-onset form with comparatively milder clinical symptoms [8, 9]. Genotype-phenotype relationship is quite clear, as type of mutation and residual sulfite oxidase activity is directly correlated with the disease severity [10]. Patients with ISOD clinically diagnosed by neuroimaging which identified multicystic leukoencephalopathy and atrophy of brain [7, 11]. ISOD patient's laboratory test were majorly identified with positive urinary sulfite test, i.e. (i) increased level of S-sulfocysteine, taurine, and thiosulfate with normal level of uric acid in urine, (ii) high level of Ssulfocysteine and taurine, normal level of uric acid and methionine and low level of cystine and homocysteine in plasma [8]. Patients with ISOD harbouring homozygous or compound heterozygous mutation in SUOX gene were identified with complete loss of sulfite oxidase activity while asymptomatic carrier individual with heterozygous mutations in SUOX gene were usually presented with $50 \%$ loss of sulfite oxidase activity $[11,12]$.

In this study, we investigated a 5-days old Chinese infant girl with ISOD. Karyotype and chromosomal microarray analysis showed no chromosomal abnormalities in the patient. Whole exome sequencing (WES) was performed for the proband. WES identified a novel 16 nucleotide deletion (c.1406_1421delCCTGGCAGGTGGCTAA) and a previously reported substitution (c.1200C > G) in exon 6 of the SUOX gene in the proband. Both of these two variants are loss-of-function variants. The proband's father and mother is carrying the substitution (c.1200C > G) and deletion

(c.1406_1421delCCTGGCAGGTGGCTAA) in a heterozygous state respectively. These two variants were 
not identified in the elder brother of the proband as well as in the 100 healthy individuals. Here, we reported the first variant of SUOX gene associated with ISOD in Chinese population.

\section{Methods}

\section{Patients and Clinical Materials}

In this study, a 5-days old Han Chinese girl with ISOD was recruited and enrolled in the Division of Maternal-Fetal Medicine, Bao'an Women and Children's Hospital, Jinan University, Shenzhen, China (Figure 1.A).

\section{Radiological, Physical and Biochemical Examination}

Ultrasonography (USG), magnetic resonance imaging (MRI) and computerized tomography (CT) were performed. Physical, biochemical and routine blood tests were performed in the proband. The proband was also tested for genetic metabolic diseases. Analysis of heme, reticulocyte and hsCRP has been done for the proband. Liver function, renal function, electrolyte and blood ammonia test were also undertaken for the proband. We also performed TroPI, myocardial enzyme and AMON test for the proband.

\section{Karyotyping and chromosomal microarray analysis (CMA)}

In the proband, standard G-banding karyotyping was performed to analyse the structure of all the chromosomes. Chromosome microarray analysis (CNA) has also been done in the proband for identifying copy number variations (CNV). CytoScan HD array (Affymetrix) was used for performing CNA. Analysis of CNA data was done by using Chromosome Analysis Suite software version 1.2.2. The copy number threshold was set at $10 \mathrm{~kb}$ and the marker count was set at $\geq 50$ [13].

\section{WES and identification of candidate variants}

Whole exome sequencing (WES) was performed for the proband. Genomic DNA of the proband was extracted from her peripheral blood according to the manufacturer's instructions. Genomic DNA of the proband was then subjected to WES. Sequences were captured by Agilent SureSelect version 6 (Agilent Technologies, Santa Clara, CA). Then, the enriched library was subjected to sequence with Illumina HighSeq 4000 platform. After that, the sequencing reads were aligned with GRCh37.p10 by using Burrows-Wheeler Aligner software (version 0.59). Next, Burrows-Wheeler aligned reads were locally aligned by using GATK Indel Realigner (broadinstitute.org/). Base quality recalibration of BurrowsWheeler aligned reads was performed by the GATK Base Recalibrator (broadinstitute.org/). Then, we identified single-nucleotide variants (SNVs) and small insertions or deletions (InDels) by using GATK Unified Genotyper (broadinstitute.org/). Lastly, annotation of the identified variants with the Consensus 
Coding Sequences Database (20130630) at the National Center for Biotechnology Information (NCBI) was performed. The quality control data of WES has been illustrated in Supplementary Table S1.

WES data were interpreted and analysed according to our previously published article [13]. Firstly, variants were selected based on their minor allele frequencies $<0.05$ in various databases (dbSNP, HapMap, 1000 Genomes Project and BGI database with 50,000 Chinese Han samples). Secondly, identified variants were functionally classified into pathogenic, likely pathogenic, VUS, likely benign and benign groups according to the variant interpretation guidelines of American College of Medical Genetics and Genomics (ACMG) [14]. Finally, the remaining variations in the proband and her unaffected were also analysed on the basis of the reference of the OMIM (https://www.omim.org) and other published resources. The data interpretation and bioinformatics analysis are schematically illustrated and described in Figure 1.B.

\section{Sanger sequencing}

Sanger sequencing was performed for the proband as well as for her parents to validate the identified variants in WES. Primer pairs were designed for the candidate loci on the basis of reference genomic sequences of the Human Genome from GenBank in NCBI. Primers were synthesized (Invitrogen, Shanghai, China) and polymerase chain reaction (PCR) has been done by using an ABI 9700 Thermal Cycler. Then, PCR products were directly sequenced by an ABI PRISM 3730 automated sequencer (Applied Biosystems, Foster City, CA, USA). Sequencing data analysis was performed by DNASTAR SeqMan (DNASTAR, Madison, Wisconsin, USA).

Sanger sequencing validated the identified heterozygous variants by WES by using the following primers: F1 5'-GCGCGGATCTCAGCATGCGCGG-3', R1 5'-GGGGTCTCCGCGGCTCCCCGG-3'; F1 5'GGCGCTAGGGTCGAAGGCGGCG-3', R1 5'-GGTCTCGGTAGGCGCGTACTGG-3' The reference sequence NM_000456 of SUOX gene was used.

\section{In silico Analysis}

In silico analysis of identified variants was performed by Mutation Taster (http://mutationtaster.org/) [15].

\section{Results}

\section{Index case}

In this study, we investigated a proband with ISOD from a nonconsanguineous Han Chinese family (Figure 1A). Proband's younger sister was died and her younger brother was phenotypically normal. Proband's parents were also phenotypically normal and asymptomatic. 
In 2009, after a successful and uneventful pregnancy, proband's mother (I-2) gave birth to a baby girl (II1). One hour after birth, she was identified with "general cyanosis" and consecutively transferred to the department of neonatology and pediatrics of our hospital for treatment. After admission, we found that the baby was unable for sucking milk and high tension of her quadrilateral muscles with spasmodic twitch. Then, we performed CT and found subarachnoid haemorrhage. Hence, we clinically diagnosed the baby girl with "neonatal hypoxic-ischemic encephalopathy with subarachnoid hemorrhage". The child died on the same day.

Two years later, in 2011, proband's mother (I-2) gave birth to a baby boy (II-2). During pregnancy, the color ultrasound was performed and showed that the posterior fossa of the fetus was $1.03 \mathrm{~cm}$. After birth, the baby had repeated intussusception. At the age of 2 months, the baby had convulsions once. The baby's head was found stable at the age of 5 months. At the age of 18 months, the baby could walk alone. We performed CT and found that the bilateral frontotemporal lobe sulcus and fissure were widened, and the brain was not fully developed. The rehabilitation treatment was carried out in the hospital. Presently, the psychomotor development of the boy is become normal.

In 2019, proband's mother (I-2) achieved her third pregnancy. Prenatal examination identified no abnormalities. At 31 weeks of gestation, B-ultrasound was performed and found no abnormalities. However, at 37+4 weeks of gestation, B-ultrasound was performed again and showed that the left lateral ventricle was $1.12 \mathrm{~cm}$ wide (Figure 2.A), the depth of cisterna magna was $1.44 \mathrm{~cm}$ (Figure 2.B), there was a cystic area about $1.61 \mathrm{~cm} \times 0.91 \mathrm{~cm}$ in size located in the midline of the brain above the anterior thalamus (Figure 2.C), and thin corpus callosum (Figure 2.D). At 38 weeks of gestation, MRI was performed and found that the posterior horn of bilateral ventricles was slightly widened, the third ventricles were enlarged and uplifted, connected with the longitudinal fissure cistern of the brain, and the possible absence of the corpus callosum was considered. There were many small and flaky abnormal signal shadows were identified in the white matter of bilateral frontotemporal lobe and bilateral basal ganglia, as well as many softened foci were found (Figure 2.E-H).

At $38+4$ weeks of gestation, a live baby girl, the proband was born through a cesarean section. The birth weight of the proband was $3.17 \mathrm{~kg}$, and the Apgar score was 10 . Soon after delivery, the proband was identified with intermittent tremor or seizures in limb. Five days after birth, we also found that the proband was presented with intermittent tremor or seizures in limb, neonatal encephalopathy, subarachnoid haemorrhage, subarachnoid cyst, dysplasia of corpus callosum, neonatal convulsion, hypotension, respiratory failure, heart failure, patent foramen ovale, hyperlactatemia, severe metabolic acidosis, hyperglycemia, hyperkalemia, moderate anemia, high atrioventricular block and complete right bundle branch block.

Combined with the prenatal MRI result and based on the clinical symptoms, the proband was immediately transferred to the neonatological department of our hospital. Emergency CT scan was performed and showed multiple hypodense lesions in bilateral, frontal and parietal cortex, symmetrical hyperdense shadows were identified in bilateral basal ganglia, dysplasia of corpus callosum, a small 
amount of subarachnoid hemorrhage, enlargement of cisterna magna, possible subarachnoid abscess and compression of cerebellar hemisphere (Figure 2. I-M).

After admission, the patient still had intermittent scream, obvious tremor or seizures in limb, decrease in oxygen saturation in blood and blood pressure under the sedative state with chloral hydrate. The proband was also sedated by the treatment with phenobarbital sodium, and the convulsion still occasionally occurred. Dopamine and dobutamine were continuously pumped to maintain normal blood pressure, while continuous positive airway pressure ventilation was used to maintain the normal blood oxygen.

The proband was tested for genetic metabolic diseases and found no abnormalities (Supplementary Table S2). The proband was identified with high level of mean corpulscular volume (MCV), mean corpuscular hemoglobin (MCH), neutrophilic granulocyte percentage (NEUT\%), immature cell percentage (IG\%), NRBC\%, NEUT, MONO\#, IG\#, NRBC\#, RDW-SD, RDW-CV and IRF as well as low level of hemoglobin concentration (HGB), lymphocytes percentage (LYMPH\%) and LFR (Supplementary Table S3). Liver function, renal function, electrolyte and blood ammonia test of the proband showed extremely high level of GGT, high level of Aspartic transaminase (AST), AST/ALT and low level of Total protein (TP) and Albumin (ALB) (Supplementary Table S4). We also performed TroPI, myocardial enzyme and AMON test for the proband and found extremely high level of Creatine Kinase (CK), Creatine kinase MB isoenzyme (CK-MB), Troponin (TroPI) and Ammonia (AMON) (Supplementary Table S5).

On the 6th day of hospitalization, the proband was identified with a sudden weak breath, the heart rate dropped to about 80 times/minute, the heart sound was low and blunt, the blood oxygen saturation dropped to about $70 \%$, the blood pressure was $47 / 12 \mathrm{mmHg}$. The patient was immediately intubated with mechanical ventilation, the resuscitation capsule was pressurized with oxygen, and no improvement was found after external chest compression, and finally the proband died.

\section{Karyotype and chromosomal microarray analyses}

Karyotyping found no abnormalities in structure of all the chromosomes in the proband (46, XX) (Figure 3.A). No pathogenic copy number variations (CNVs) were identified in all the chromosomes of the proband by CAN (Figure 3.B).

\section{WES and Sanger sequencing identified a novel variant in SUOX gene}

WES was performed for the proband. WES identified a novel 16 nucleotide deletion (c.1406_1421delCCTGGCAGGTGGCTAA) and a previously reported substitution (c.1200C>G) in exon 6 of the SUOX gene in the proband (Figure 4). The novel 16 nucleotide deletion

(c.1406_1421delCCTGGCAGGTGGCTAA) leads to a frameshift (p.Thr469Serfs*20) followed by premature termination of translation which finally results into the formation of a truncated sulfite oxidase of 488 
amino acids instead of the wild type sulfite oxidase of 545 amino acids. The substitution (c. $1200 \mathrm{C}>\mathrm{G}$ ) also leads to a premature stop codon (p.Y400*) followed by the formation of a truncated sulfite oxidase of 399 amino acids instead of the wild type sulfite oxidase of 545 amino acids. Hence, both the variants are loss-of-function variants. The proband's father and mother is carrying the substitution (c.1200C $>G$ ) and deletion (c.1406_1421delCCTGGCAGGTGGCTAA) in a heterozygous state respectively (Figure 4). These two variants were not identified in the elder brother of the proband as well as in the 100 healthy individuals. This heterozygous novel 16 nucleotide deletion (c.1406_1421delCCTGGCAGGTGGCTAA) also not present in the Human Gene Variant database (HGMD, www.hgmd.cf.ac.uk/), Online Mendelian Inheritance in Man (MIM, (https://www.omim.org). These two heterozygous variants are not found in BGl's database, consisting of $\sim 50,000$ Chinese Han samples. We also did not find these two variants in ExAC, gnomAD, dbSNP and 1000 Genome Database. Our present study identified the first variant in SUOX gene associated with ISOD in Chinese population. In this study, we also described the importance of WES as a potential sequencing technology for identifying candidate variant in the SUOX associated ISOD patients.

\section{In silico Analysis}

These two variants (c.1406_1421 delCCTGGCAGGTGGCTAA, p.Thr469Serfs*20; c.1200C>G, p.Tyr400*) were predicted as "disease causing" by Mutation Taster (http://mutationtaster.org/) [15].

\section{Discussion}

In this study, we investigated a 5-days old Chinese girl child who was presented with Classic ISOD. Karyotype and chromosomal microarray analysis identified no chromosomal abnormalities in the patient. Whole exome sequencing identified a novel 16 nucleotide deletion

(c.1406_1421 delCCTGGCAGGTGGCTAA) and a previously reported substitution (c.1200C > G) in exon 6 of the SUOX gene in the proband. Both of these variants lead to the formation of truncated sulfite oxidase. Hence, both the variants are loss-of-function variants. The proband's father and mother is carrying the substitution (c.1200C > G) and deletion (c.1406_1421delCCTGGCAGGTGGCTAA) in a heterozygous state respectively. These two variants were not identified in the elder brother of the proband. According to the variant interpretation guidelines of American College of Medical Genetics and Genomics (ACMG), this variant is categorized as "likely pathogenic" variant [14].

The proband inherited the heterozygous substitution (c.1200C $>\mathrm{G}$ ) from her father. This variant was identified in homozygous state in two patients with ISOD in Taiwan [16, 17]. In 2012, Huang et al., investigated a female new born presented with high-pitched cry, tonic movements of four limbs, seizures without desaturation, decreased level of total homocysteine in plasma, diffuse cerebral atrophy and persistent edema in the left occipital, posterior temporal, and parietal lobes [16]. Proband's parents were healthy and nonconsanguineous without any family history. The proband was born through an 
unremarkable pregnancy. The proband was not identified with any obvious dysmorphic features with normal uric acid level [16].

In 2017, Lee et al., reported a female new born clinically diagnosed with ISOD from Taiwan. The proband was born after an uneventful pregnancy. Proband's parents were truly non-consanguineous without any family history. The proband was manifested with difficulty in feeding and prolonged feeding time with poor sucking power. The proband was also presented with seizures with bicycling of legs, alternating myoclonic seizures with rhythmic jerking over limbs, and high-pitched irritable cry. Proband's brain MRI showed ventricular dilatation, left frontal and temporal areas with cystic lesions, high T2 signal intensity of the bilateral cerebral cortex and globus pallidi. The proband was identified with normal level of bicarbonate, ammonia, plasma uric acid, amino acids, acylcarnitine, and urinary organic acids with absence of sufites in urine as well as a very low level of plasma cysteine [17].

Generally, ISOD is manifested with axial and peripheral hypotonia with movement abnormality, seizures, spasticity, microcephaly, intellectual deficit, difficulties in feeding, severe psychomotor retardation and ectopia lentis or dislocation of lens with an early infantile or neonatal age of onset. Axial and peripheral hypertonia are the most common phenotype among ISOD patients. Pharmaco-resistant seizures with abnormalities in muscle tone and movements were usually identified in most of the ISOD patients. Gradual and progressive difficulties in feeding was also found in many ISOD patients. According to neuropathological symptoms, it is impossible to distinguish between ISOD patients and the patients with severe perinatal asphyxia $[18,19]$. Zaki et al., strongly recommended to include ISOD in the new-born differential clinical diagnosis with neonatal convulsion, seizures, abnormalities in movement and EEG test result [20]. Previous studies showed that typical neuropathological (progressive changes in white matter, atrophy of cerebrum and cerebellum, cystic leukomalacia and ventriculomegaly) features of ISOD patients usually identified by neuroimaging (CT or MRI) [21, 22].

Till date, no curative treatment has been developed for the patients with ISOD. In human, catabolism of cysteine is the major source of sulfite, so, late-onset ISOD patients with milder clinical symptoms usually recommended with low-methionine or low-cysteine diet [8,23, 24]. Previous study reported that two lateonset ISOD patients with mild clinical phenotype has been treated with low-methionine and low-cysteine diet and showed gradual progress in psychomotor development without neurological deterioration [23]. However, ISOD patients with early or neonatal age of onset usually showed lethal outcome [25].

Sulfite oxidase is a molybdenum dependent enzyme. Pre-sulfite oxidase translocated from the cytosol to the outer mitochondrial membrane through the $\mathrm{N}$-terminal ladder peptide which subsequently anchored on the inner mitochondrial membrane [26]. Inner membrane peptidase (IMP) cleaved the N-terminal ladder peptide and the $\mathrm{C}$-terminal fragment of sulfite oxidase has been released into the intermembrane space (IMS). Sulfite oxidase catalytically activated after binding with heme and molybdenum cofactor followed by homodimerization $[21,26]$.

Till now, only 29 germline variants of SUOX have been identified to be associated with ISOD. Among 29 reported variants, 19 variants are missense, 3 variants are nonsense and 6 variants are deletion which 
result into frameshift $[3,7,8,9,11,20,27-32]$. One deletion leads to loss of the wild type stop codon and followed by formation of a prolonged protein [25].

ISOD is a very rare and extremely heterogenous disorder in terms of both genotype and phenotype. However, due to extreme genotypic and phenotypic heterogeneity among patients with ISOD, identifying candidate gene and disease-causing mutation is a great challenge. Genetic screening of ISOD patients by performing next generation sequencing, either single gene sequencing or sequencing of a panel of genes are not always able to identify the candidate variants underlying the disease phenotype in ISOD patients. In order to overcome these disadvantages, whole exome sequencing, specially WES is the most significant and recent sequencing technologies for identifying the candidate gene and disease-causing variants in patients with ISOD. WES is the most sophisticated and advanced technique considering for identifying the candidate gene variants. allowing clinicians for making timely and proper clinical diagnosis [33-35]. In conclusion, our present study not only report the first variant of SUOX gene in a patient with ISOD in Chinese population, but also describe the importance and application of WES as a potential high throughput sequencing technology for molecular genetic analysis for the patients with ISOD.

\section{Conclusion}

Here, we report the first Chinese patient identified with extremely rare ISOD. Our present study expanded the mutation spectrum of SUOX gene associated with ISOD. Here, we also illustrated the significance of whole exome sequencing for identifying the candidate gene and disease-causing variants in patients with ISOD. This study also helped the clinicians for making proper clinical diagnosis of the ISOD patients with germline mutation in SUOX gene.

\section{Abbreviations}

ISOD: Isolated sulfite oxidase deficiency; MoCoD: Molybdenum cofactor deficiency; MRI: Magnetic resonance imaging; MRS: Magnetic resonance spectroscopy; SO: Sulfite oxidase

\section{Declarations}

\section{Acknowledgements}

We are thankful to the proband and all the family members for participating in our study.

\section{Funding}

We also thankful to the Guangdong Enterprise Key Laboratory of Human Disease Genomics (2011A060906007). 


\section{Availability of data materials}

Study data for the primary analyses presented in this manuscript are available upon reasonable request from the corresponding author.

\section{Authors' contributions}

Conceptualization: S.B., H.W. and R.Z. Data curation: R.Z., Y.H., Y.X. and J.Q. Formal analysis: Y.W., S.K.D., C.L. Investigation: S.B., H.W. and R.Z. Methodology: Y.W., S.K.D., Y.H., J.Q. and C.L. Project administration: S.B. and H.W. Resources: S.B. and H.W. Super vision: S.B. and H.W. Writing-original draft: S.B., H.W., R.Z., C.L. and S.K.D.

\section{Competing interests}

The authors declare that they have no competing interests.

\section{Consent for publication}

Not applicable.

\section{Ethics approval and consent to participate}

The ethics committee of the Bao'an Women and Children's Hospital, Jinan University, Shenzhen, China, approved the study, according to the recommendations of the Declaration of Helsinki. Written informed consent were obtained from all the participant of this study. The proband and his family members provided written informed consent for the publication of the patient's identifiable information.

\section{References}

1. Schwarz G, Mendel RR, Ribbe MW. Molybdenum cofactors, enzymes and pathways. Nature. 2009;460(7257):839-47.

2. Brown GK, Scholem RD, Croll HB, Wraith JE, McGill JJ. Sulfite oxidase deficiency: clinical, neuroradiologic, and biochemical features in two new patients. Neurology. 1989;39(2 Pt 1):252-7.

3. Chen LW, Tsai YS, Huang CC. Prenatal multicystic encephalopathy in isolated sulfite oxidase deficiency with a novel mutation. Pediatr Neurol. 2014;51(1):181-2.

4. Lee HF, Mak BSC, Chi CS, Tsai CR, Chen CH, Shu SG. A novel mutation in neonatal isolated sulphite oxidase deficiency. Neuropediatrics. 2002;33(4):174-9. 
5. Carmi-Nawi N, Malinger G, Mandel H, Ichida K, Lerman-Sagie T, Lev D. Prenatal brain disruption in molybdenum cofactor deficiency. J Child Neurol. 2011;26(4):460-4.

6. Sass JO, Nakanishi T, Sato T, Shimizu A. New approaches towards laboratory diagnosis of isolated sulphite oxidase deficiency. Ann Clin Biochem. 2004;41(Pt 2):157-9.

7. Tan WH, Eichler FS, Hoda S, Lee MS, Baris H, Hanley CA, et al. Isolated sulphite oxidase deficiency: a case report with a novel mutation and review of the literature. Pediatrics. 2005;116(3):757-66.

8. Rocha S, Ferreira AC, Dias Al, Vieira JP, Sequeira S. Sulfite oxidase deficiency-an unusual late and mild presentation. Brain Dev. 2014;36(2):176-9.

9. Salih MA, Bosley TM, Alorainy IA, Sabry MA, Rashed MS, Al-Yamani EA, et al. Preimplantation genetic diagnosis in isolated sulphite oxidase deficiency. Can J Neurol Sci. 2013;40(1):109-12.

10. Schwahn BC, Van Spronsen FJ, Belaidi AA, Bowhay S, Christodoulou J, Derks TG, et al. Efficacy and safety of cyclic pyranopterin monophosphate substitution in severe molybdenum cofactor deficiency type a: a prospective cohort study. Lancet. 2015;386(10007):1955-63.

11. Johnson JL, Coyne KE, Garrett RM, Zabot MT, Dorche C, Kisker C, et al. Isolated sulfite oxidase deficiency: identification of 12 novel SUOX mutations in 10 patients. Hum Mutat. 2002;20(1):74.

12. Holder JL Jr, Agadi S, Reese W, Rehder C, Quach MM. Infantile spasms and hyperekplexia associated with isolated sulfite oxidase deficiency. JAMA Neurol. 2014;71(6):782-4.

13. Zhang R, Chen S, Han P, Chen F, Kuang S, Meng Z, et al. Whole exome sequencing identified a homozygous novel variant in CEP290 gene causes Meckel syndrome. J Cell Mol Med. 2020;24(2):1906-16.

14. Richards S, Aziz N, Bale S, Bick D, Das S, Gastier-Foster J, et al. Standards and guidelines for the interpretation of sequence variants: a joint consensus recommendation of the American College of Medical Genetics and Genomics and the Association for Molecular Pathology. Genet Med. 2015;17(5):405-24.

15. Schwarz JM, Cooper DN, Schuelke M, Seelow D. MutationTaster2: mutation prediction for the deepsequencing age. Nat Methods. 2014;11(4):361-2.

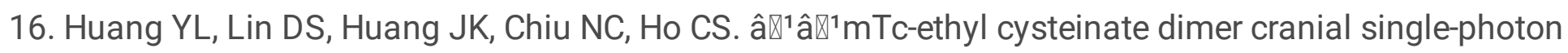
emission computed tomography and serial cranial magnetic resonance imaging in a girl with isolated sulfite oxidase deficiency. Pediatr Neurol. 2012;47(1):44-6.

17. Lee HF, Chi CS, Tsai CR, Chen HC, Lee IC. Prenatal brain disruption in isolated sulfite oxidase deficiency. Orphanet J Rare Dis. 2017;12(1):115.

18. Hobson E, Thomas S, Crofton PM, Murray AD, Dean J, Lloyd D. Isolated sulphite oxidase deficiency mimics the features of hypoxic ischaemic encephalopathy. Eur J Pediatr. 2005;164(11):655-9.

19. Tyagarajan SK, Fritschy JM. Gephyrin: a master regulator of neuronal function? Nat Rev Neurosci. 2014;15(3):141-56.

20. Zaki MS, Selim L, El-Bassyouni HT, Mahmoud YI, Mahmoud I, Ismail S, et al. Molybdenum cofactor and isolated sulphite oxidase deficiencies: clinical and molecular spectrum among Egyptian 
patients. Eur J Paediatr Neurol. 2016;20(5):714-22.

21. Klein JM, Schwarz G. Cofactor-dependent maturation of mammalian sulfite oxidase links two mitochondrial import pathways. J Cell Sci. 2012;125(Pt 20):4876-85.

22. Terao M, Romão MJ, Leimkühler $S$, Bolis $M$, Fratelli $M$, Coelho $C$, et al. Structure and function of mammalian aldehyde oxidases. Arch Toxicol. 2016;90(4):753-80.

23. Touati G, Rusthoven E, Depondt E, Dorche C, Duran M, Heron B, et al. Dietary therapy in two patients with a mild form of sulphite oxidase deficiency. Evidence for clinical and biological improvement. J Inherit Metab Dis. 2000;23(1):45-53.

24. Del M, Burlina AP, Sass J, Beermann F, Zanco C, Cazzorla C, et al. Metabolic stroke in a late-onset form of isolated sulfite oxidase deficiency. Mol Genet Metab. 2013;108(4):263-6.

25. Johnson JL, Rajagopalan KV, Renier WO, Van der Burgt I, Ruitenbeek W. Isolated sulfite oxidase deficiency: mutation analysis and DNA-based prenatal diagnosis. Prenat Diagn. 2002;22(5):433-6.

26. Hahn A, Engelhard C, Reschke S, Teutloff C, Bittl R, Leimkühler S, Risse T. Structural insights into the incorporation of the Mo cofactor into sulfite oxidase from site-directed spin labeling. Angew Chemie. 2015;54:11865-9.

27. Bastarache L, Hughey JJ, Hebbring S, Marlo J, Zhao W, Ho WT, et al. Phenotype risk scores identify patients with unrecognized Mendelian disease patterns. Science. 2018;359(6381):1233-9.

28. Brumaru D, Guerin E, Voegeli AC, Eyer D, Maitre M. A compound heterozygote case of isolated sulfite oxidase deficiency. Mol Genet Metab Rep. 2017;12:99-102.

29. Kisker C, Schindelin H, Pacheco A, Wehbi W, Garrett RM, Rajagopalan KV, et al. Molecular basis of sulfite oxidase deficiency from the structure of sulfite oxidase. Cell. 1997;91(7):973-83.

30. Bi C, Wu J, Jiang T, Liu Q, Cai W, Yu P, et al. Mutations of ANK3 identified by exome sequencing are associated with autism susceptibility. Hum Mutat. 2012;33(12):1635-8.

31. Seidahmed MZ, Alyamani EA, Rashed MS, Saadallah AA, Abdelbasit OB, Shaheed MM, et al. Total truncation of the molybdopterin/dimerization domains of SUOX protein in an Arab family with isolated sulfite oxidase deficiency. Am J Med Genet A. 2005;136(2):205-9.

32. Rupar CA, Gillett J, Gordon BA, Ramsay DA, Johnson JL, Garrett RM, et al. Isolated sulfite oxidase deficiency. Neuropediatrics. 1996;27(6):299-304.

33. Dai Y, Liang S, Dong X, Zhao Y, Ren H, Guan Y, et al. Whole exome sequencing identified a novel DAG1 mutation in a patient with rare, mild and late age of onset muscular dystrophydystroglycanopathy. J Cell Mol Med. 2019;23(2):811-8.

34. Ng SB, Buckingham KJ, Lee C, Bigham AW, Tabor HK, Dent KM, et al. Exome sequencing identifies the cause of a mendelian disorder. Nat Genet. 2010;42(1):30-5.

35. Zheng Y, Xu J, Liang S, Lin D, Banerjee S. Whole Exome Sequencing Identified a Novel Heterozygous Mutation in HMBS Gene in a Chinese Patient with Acute Intermittent Porphyria with Rare Type of Mild Anemia. Front Genet. 2018;9:129. 
A I

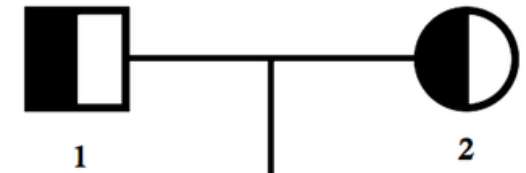

c.1200C $>$ G/WT

c.1406_1421delCCTGGCAGGTGGCTAA/WT

II

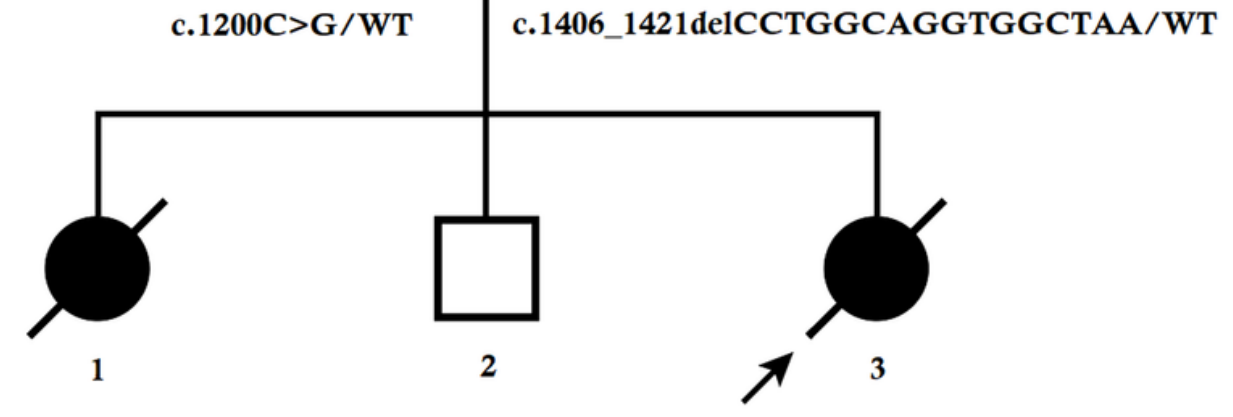

WT/WT c.1200C>G/1406_1421delCCTGGCAGGTGGCTAA

$\mathrm{B}$
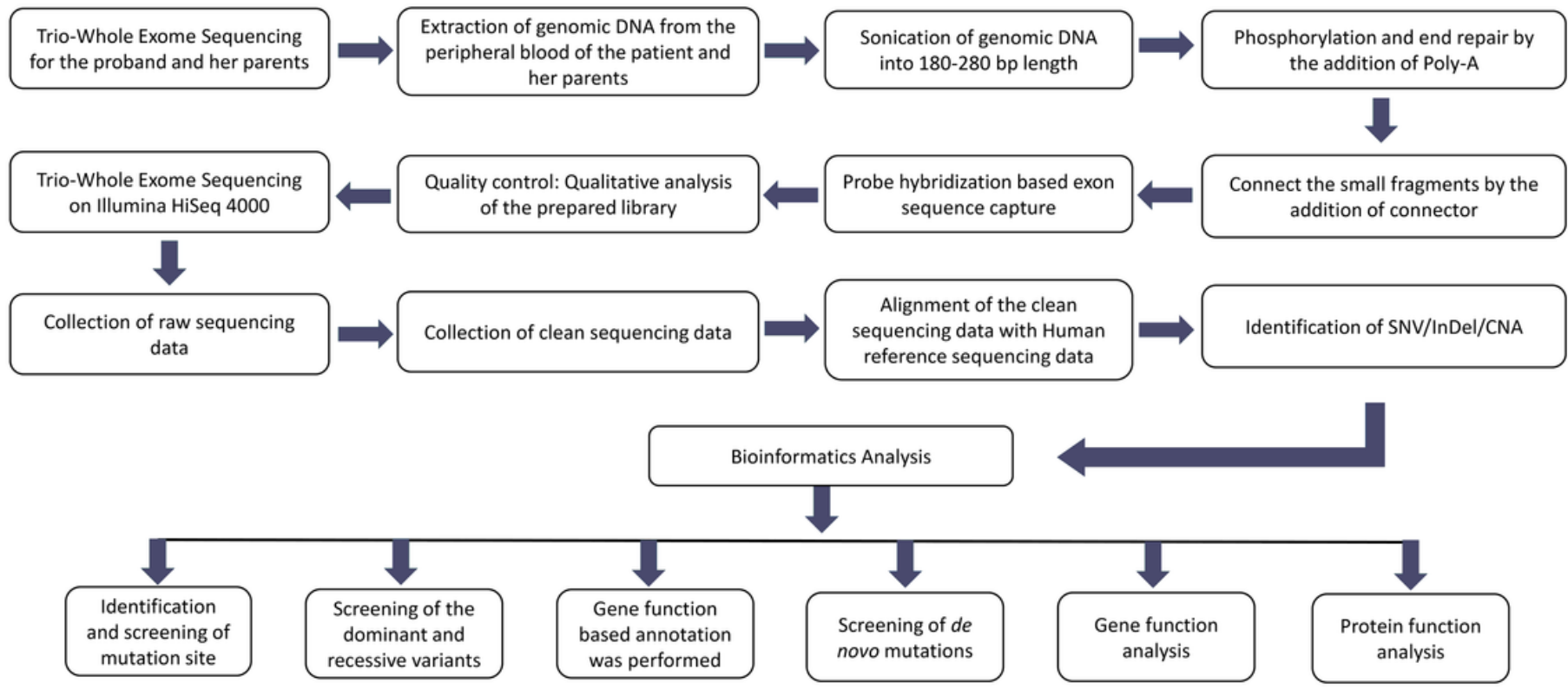

\section{Figure 1}

A. Pedigree of the Chinese family with ISOD. Squares and circles denoted males and females respectively. Individuals labelled with a solidus were deceased. Roman numerals indicate generations. Arrow indicates the proband (II-3). B. Schematic presentation of the detailed data interpretation pipeline. 

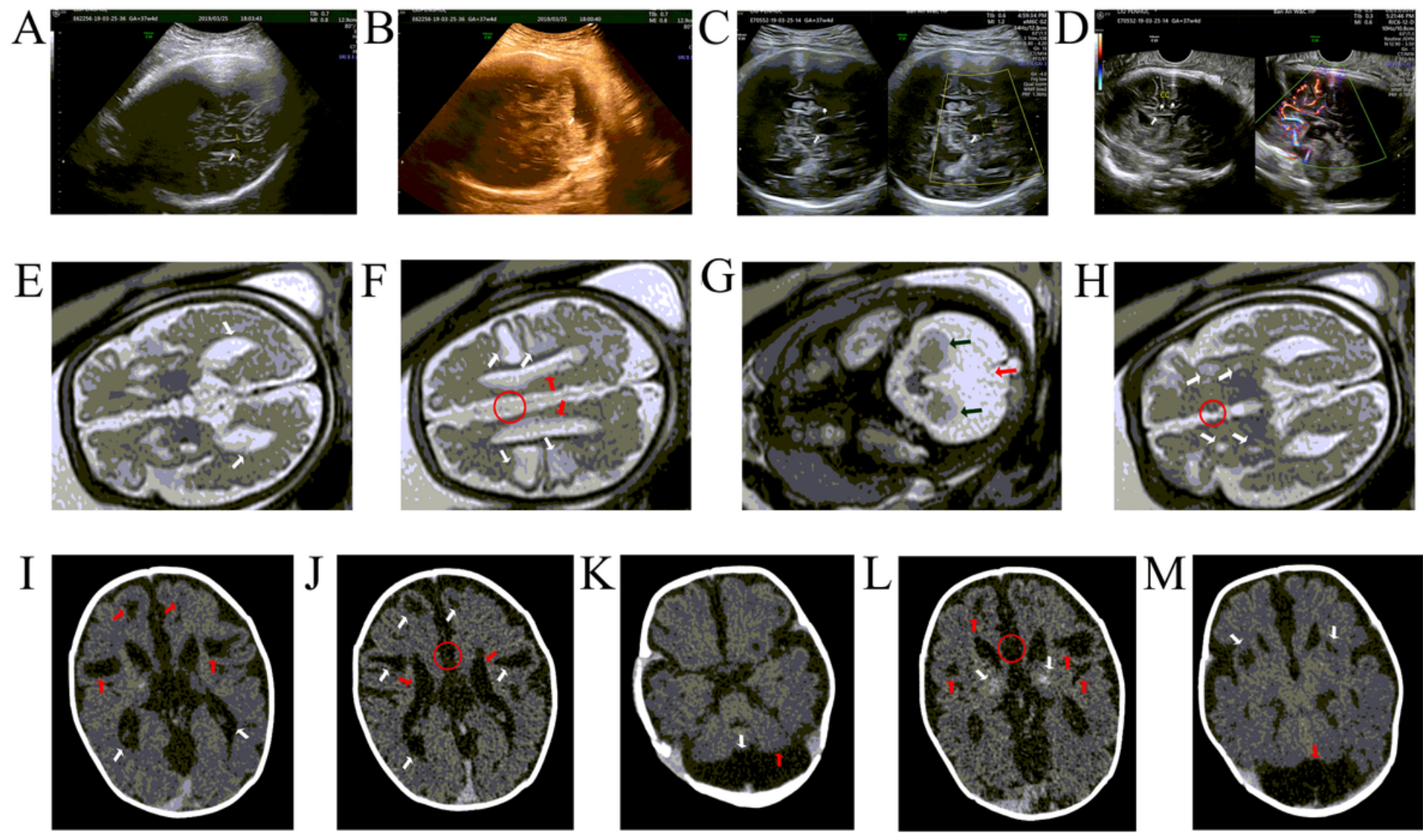

\section{Figure 2}

A-D. Ultrasonography examination. A. The width of the left lateral ventricle was $1.12 \mathrm{~cm}$ (white arrow). B. The depth of cisterna magna was $1.44 \mathrm{~cm}$ (white arrow). C. A $1.6^{\star} 0.9 \mathrm{~cm}$ cystic structure was identified in the midline falx. D. The agenesis of the corpus callosum and the posterior part of the pericallosal artery not showed completely. E-H. Magnetic Resonance Imaging (MRI) examination. E. The axial T2WI images found that the anterior horns of the bilateral ventricle were slightly enlarged (white arrow). The width of them were $11.2 \mathrm{~mm}$ in left and $10.2 \mathrm{~mm}$ in right. F: The axial T2Wl images showed several flake-shape foci with T2WI high signal intensities (white arrow) symmetrically demonstrated in the white matter of bilateral frontal and parietal lobes. The longitudinal fissure of the brain was enlarged, and the knee of the corpus callosum was not detected (red circle). The bilateral ventricles were enlarged and parallel with the length of the midline (red arrow). G. The axial T2WI images identified that the cisterna magna was severely enlarged (red arrow) with T2WI high signal intensity. The compressed skull showed in the corresponding part. The bilateral cerebellar hemispheres were small (black arrow). $\mathrm{H}$. The axial T2WI images demonstrated several round-shape foci with T2WI high-signal intensity (white arrow) in the white matter of the right frontal lobe, left basal ganglia and bilateral insular lobes. The longitudinal fissure of the brain was enlarged, and the knee of the corpus callosum was not detected (red circle). I-M. Computed tomography (CT) examination. I. The posterior portions of bilateral ventricle were identified with slight ventriculomegaly (white arrow). The bilateral frontal lobe showed the patchy low-density shadow (red arrow). J. Several patchy low densities (white arrows) symmetrically demonstrated in the white matter of 
bilateral frontal and parietal lobes. The longitudinal fissure of the brain was enlarged, and the knee of the corpus callosum was not detected (red circle). The distance between the bilateral ventricles was increased and parallel with the length of the midline (red arrow). K. The cisterna magna was significantly enlarged (white arrow), and the bilateral cerebellar hemispheres were small (red arrow). L. The bilateral basal ganglia showed slightly high density (white arrow). Several patchy low-density shadows were identified under the bilateral frontal and parietal cortex (red arrow); the longitudinal fissure was enlarged (red circle). M. The bilateral insular lobes showed patchy and low-density lesion (white arrow). The cisterna magna was severely enlarged (red arrow).
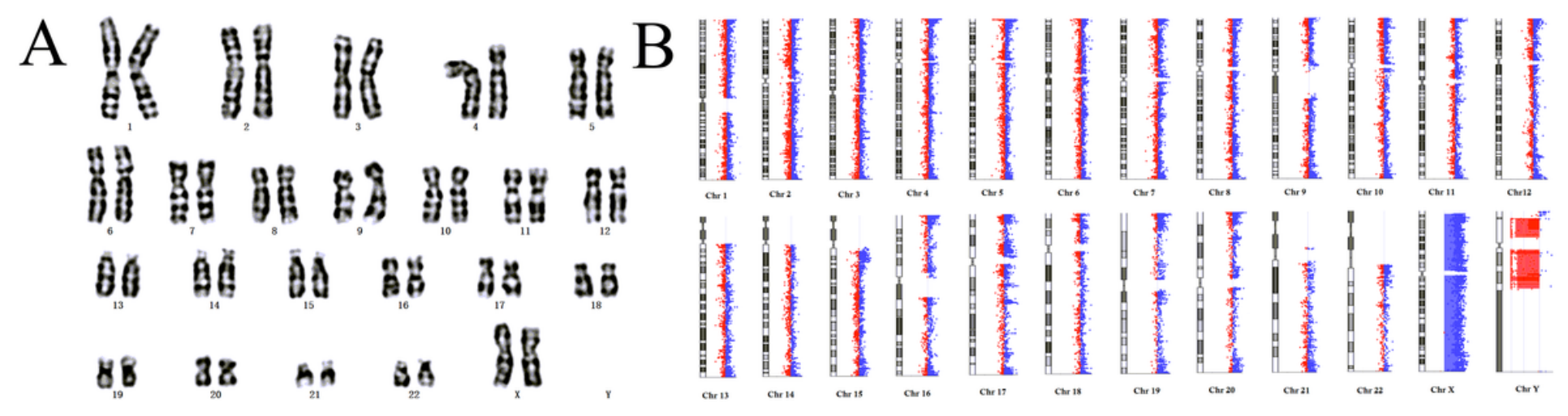

\section{Figure 3}

A. Karyotype of the patient. Proband's karyotype was normal. B. Chromosome Microarray Analysis. Chromosome microarray analysis found no copy number variation (CNV).
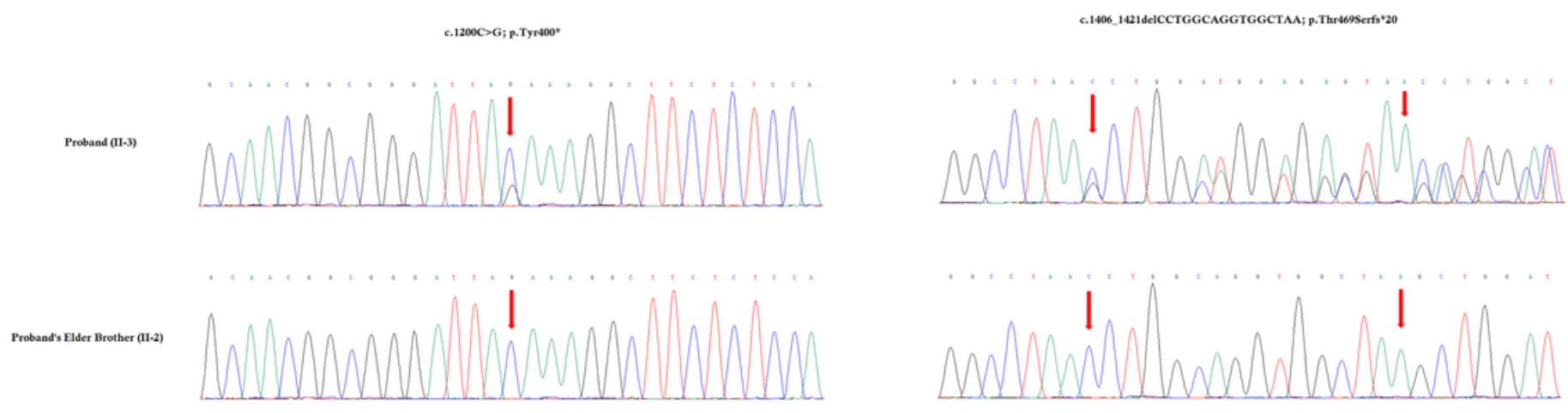

Proband's father (1-1)
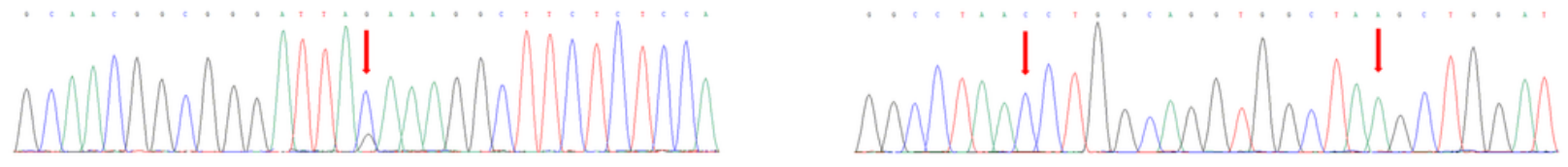

Proband's mother (-2)
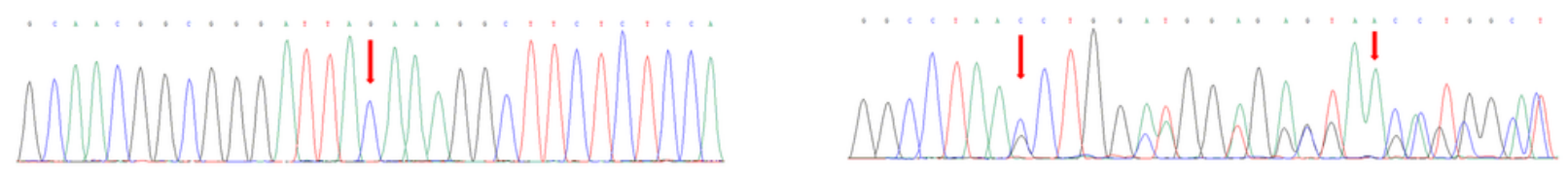

Figure 4 
Partial DNA sequences in the SUOX gene by Sanger sequencing of the family. The reference sequence NM_000456 of SUOX gene was used.

\section{Supplementary Files}

This is a list of supplementary files associated with this preprint. Click to download.

- SupplementaryTableS5.docx

- SupplementaryTableS4.docx

- SupplementaryTableS3.docx

- SupplementaryTableS2.docx

- SupplementaryTableS1.docx 